\title{
Social Research and Implementation Path Analysis of Safety Helmets Wearing by E-bicycle Riders
}

\author{
Siyi Jiang ${ }^{1, *}$ Qi Liu ${ }^{1}$ \\ ${ }^{1}$ Nanjing University of Finance \& Economics Hongshan College, Nanjing, Jiangsu 210009, China \\ *Corresponding author. Email: 2874952928@qq.com
}

\begin{abstract}
The paper aims to explore the effective implementation path of the policy that electric bicycle riders need to wear helmets. Local laws and regulations issued by the provinces, especially Jiangsu Province, and the overall implementation status since the introduction of the policy were collected in the paper. Online questionnaires and offline visits focusing on the masses and helmet products are employed to investigate the basic intentions of the masses, the changes in the helmet market and the shortcomings of existing helmets, etc., and explore effective paths based on this. Approaches to improving local laws and regulations, strengthening community propaganda, innovating staff safety and security functions, optimizing helmet structure, and promoting smart helmet are discovered to facilitate the effective implementation of the policy.
\end{abstract}

Keywords: Electric bicycle, Helmet, Intellectualization, Social research, Implementation path.

\section{INTRODUCTION}

On April 21, 2020, the Traffic Management Bureau of the Ministry of Public Security issued a notice that starting from June 1, 2020, the security guard operation of "One Helmet and One Belt (Safety helmet for people riding motorcycles and electric vehicles, and vehicle seat belt)" will be carried nationwide. As a result, the increasing number of army helmets can be seen at street intersections during morning and evening rush hours. The research and implementation path analysis in this paper is based on the security guard operation of the Traffic Management Bureau of the Ministry of Public Security and Measures of Jiangsu Province for the Administration of Electric Bicycles. Paths to promote the effective implementation of the policy will be explored to facilitate social civilization and safe riding of ebikes.

*Fund: This paper is the final result of Undergraduate Innovation and Entrepreneurship Training Program of Jiangsu Province in 2020: "Social Research and Implementation Path Analysis of Safety Helmets Wearing by E-bicycle Riders" (Project No.: 202013990010Y).

\section{RESEARCH BACKGROUND}

\subsection{Local Laws and Regulations}

"Riders of e-bikes should wear helmets" was written into law after Measures of Jiangsu Province for the Administration of Electric Bicycles was released on May 15, 2020. At the same time, the Traffic Management Bureau of the Ministry of Public Security has conducted "One Helmet and One Belt" across the country. Starting from June 1, 2020, "the drivers and riders of motorcycles and electric bicycles who do not wear safety helmets and riders who do not use safety belts are inspected and corrected according to law". As of October 1, 2020, a total of 23 local regulations have stipulated the wearing of helmets on e-bikes. Measures of Jiangsu Province for the Administration of Electric Bicycles requires enterprises that adopt electric bicycles to engage in express delivery and takeout business activities to equip their electric bicycle riders with safety helmets, and purchase third-party liability insurance, personal accident injury insurance and other relevant insurance according to their needs [1: 45]. Depending on the type of administrative punishment, the vast majority are warnings or fines. The maximum fine is set at 50 yuan. 
But not all local laws are mandatory. Some regions mention helmet wearing in specific laws for e-bikes or non-motor vehicles, while others prefer to promote local civilization, with the former advocating compulsory execution and the latter positive guiding.

\subsection{Analysis of Policy Rationality}

The progress of social civilization indicates that cars are no longer the best choice for travel. With time, geography and other factors permitting, shared bikes, electric bikes and electric bicycles have become popular with everyone. Such approach is more convenient while protecting the environment.

Overall, the number of e-bikes in China has been on the rise in recent years, with more than 67 million in Jiangsu and Zhejiang provinces alone, which also explains the frequent occurrence of electric vehicle accidents. Multi-source flow model is applied in this paper to further analyze this policy. [2]

The model involves three sources: problem stream, policy stream and political stream, whose convergence mainly benefits from policies and in turn prompts the introduction of new policies.

\subsubsection{Policy Window}

According to statistics, the number of traffic accidents nationwide in 2019 was 247,646, an increase of 2,709 over 2018, or 11.1 percent year on year. The number of traffic accidents in Jiangsu Province was 12,414 , and the death rate of electric bicycle accidents was $51.9 \%$. Despite previous regulations on a range of violations, such as running red lights, there has been a lack of strong legal restrictions on wearing safety helmets while riding e-bikes, resulting in half of the deaths caused by e-bike accidents nationwide.

\subsubsection{Problem Stream}

Regulations on the age of e-bike riders were introduced in China in 2018. Technical Specifications for Safety of Electric Bicycle was issued and implemented on April 15, 2019, focusing on regulating the production of safe electric vehicles, the classification of electric vehicles, and rules for carrying people in electric vehicles. To some extent, this reduces the traffic safety accidents caused by substandard production of electric vehicles in China. However, these regulations emphasized electric vehicle itself over public riding protection measures, such as wearing safety helmets. As a result, most people are not in the habit of wearing safety helmets. According to the statistics of European Union in 2001 and 2002, more than $70 \%$ of the people who die from driving non-motor vehicles have craniocerebral injury, and more than $50 \%$ are caused by craniocerebral injury. Therefore, safety helmet plays an essential role.

\subsubsection{Policy Stream}

Measures of Jiangsu Province for the Administration of Electric Bicycles came into force on May 15, 2020, serving as a guiding document to strengthen road traffic safety construction in Jiangsu Province. In order to strengthen the public's awareness of self-protection and encourage the public to develop the good habit of abiding by road safety laws and regulations, the government has made unremitting efforts to explore and amend relevant regulations. According to the second survey, $76.83 \%$ of respondents from Zhenjiang and Nanjing supported a 20-50 yuan fine for violators, with only $4.27 \%$ believing that the penalty was unreasonable, $10.73 \%$ that it was too benignant, and $8.54 \%$ that it was too heavy. Therefore, on the whole, most people accept this policy.

It should be noted that there is still room for improvement in this policy. The second survey revealed that $58.54 \%$ thought wearing helmets was uncomfortable, and $82.32 \%$ hoped that the internal structure design of helmets could be improved to make them more comfortable and safe. Therefore, whether the helmet is comfortable or not will largely determine whether the policy can be effectively implemented. In addition, while supervising the implementation of the policy, the public should also be informed about the correct way to wear helmets and improve the quality of helmets. [3] In this way, the important role of the helmet can be maximized in the event of an accidental injury. The results showed that most people were unclear about how to wear a helmet correctly.

\subsubsection{Political Stream}

Public mood is the key here. Before the policy, the public did not pay enough attention to wearing safety helmets while riding e-bikes. Even though most people understand the importance of wearing safety helmets and support the new rules, they are still taking chances in the absence of enforcement. 


\section{AN ANALYSIS OF HELMET WEARING POLICIES FOR ELECTRIC BICYCLES IN CHINA: A CASE STUDY OF NANJING, JIANGSU PROVINCE}

\subsection{Overall Implementation Status}

One week after the new regulation came into effect, more than $80 \%$ of cyclists in the main urban areas were wearing helmets. However, the implementation of the new regulation varies among regions, with a few drivers still taking chances. On July 27, a third-party agency conducted an evaluation of wearing helmets on electric bicycles in six districts of the main city. Among the 16 points randomly selected, Jiangdong Middle Road intersection of Xinglong Street had the highest wearing rate, reaching $98.44 \%$, and Guojiashan intersection of Central North Road had the lowest wearing rate, also reaching $90.2 \%$.

\subsection{Comparative Analysis of Policy Before and After}

After the implementation of the policy, the helmet wearing situation of electric bicycle drivers has improved significantly. Since July 1, the city's traffic police have punished a total of 12,000 illegal acts of driving and riding electric bicycles without wearing helmets on the spot, and the number of onsite penalties in a single day is generally on the decline. Compared with the number of registered education for not wearing helmets in the same period in June, the number has decreased by $67 \%$ from the previous month. Through big data analysis, Nanjing's traffic management department found that compared with the first ten days of April this year, the number of accidents involving electric bikes and the number of injured people in the city decreased by $44.5 \%$ and $47.3 \%$ respectively.

\section{ANALYSIS OF SOCIAL RESEARCH RESULTS}

\subsection{The Basic Intention of the Masses}

According to the results of the second sampling survey, $59.15 \%$ of the people understand Measures of Jiangsu Province for the Administration of Electric Bicycles, $82.95 \%$ support wearing helmets while riding electric bicycles, and $76.83 \%$ support the 20-50 yuan fine in the regulation. Although the vast majority of people expressed their understanding and support for this policy, they still took chances in the actual action.

\subsection{Degree of Dissemination}

Since the implementation of the policy, Nanjing police have simultaneously launched the helmet donation activities for public welfare: "I wear a helmet, I go first, advocating a new fashion in cycling", in 12 districts and 15 venues. More than 100 sets of propaganda equipment have been distributed, 1,000 building screens and banners have been laid out, one million propaganda pages have been printed, and 10 million text messages have been sent. In addition, activities such as juvenile traffic police teams, the first class of the new term, and small hands holding big hands have been carried out in schools, so as to foster awareness of wearing helmets. "The power of the media was utilized to make and release educational videos of 'Lucky Helmets with You', and activities such as Helmets for free via Douyin Live, knowledge contest with prizes of A Complimentary Helmet for Studying the Law, and lottery for free helmet 'Lucky Helmets with You' were launched. More than 20,000 helmets were donated to the public successively." Zeng Celi, a police officer in the propaganda room of the Traffic Police Division of the municipal public security bureau, said so.

In addition, the proper way to wear a helmet has been neglected. People are used to wearing the helmet in a way that makes them comfortable, which often leads to the failure of the helmet to function effectively. According to the second sample survey, most people were unable to accurately specify the location of the helmet's elastic adjuster, the correct position of the ears and the length of the jaw socket for the helmet to be properly worn.

\subsection{The Change of Helmet Market in China}

Product prices tend to fluctuate with market demand, policy changes, product costs, consumer psychology and other factors. Since the implementation of the policy, the helmet market has enjoyed an unprecedented "spring". [4] People buy helmets for a variety of reasons, making helmet prices go up for a period of time, and the profits of upstream companies increase. Since then, the number of competitors has brought helmet prices down, but they are still up compared to a few years ago. In order to prevent the incongruence of helmet 
market, some places gradually changed the nature of the policy from mandatory to guiding.

The survey results show that $31.78 \%$ of people accept helmets worth 80-130 yuan, $26.96 \%$ accept helmets worth more than 130 yuan, and $20.16 \%$ accept helmets worth less than 50 yuan. The main purchasing channels were online shopping and physical stores. Helmets given by car buyers and insurance companies accounted for $12.8 \%$ and $2.44 \%$, respectively.

\subsection{Shortcomings of Existing Helmets}

In the second and third sample surveys, only $7.75 \%$ of people thought their helmet was comfortable. The overwhelming majority felt the helmet wearing experience was average or even very bad, $82.32 \%$ of them hoped to improve the internal structure of the helmet to make it more comfortable to wear. (See "Figure 1" and "Figure 2")

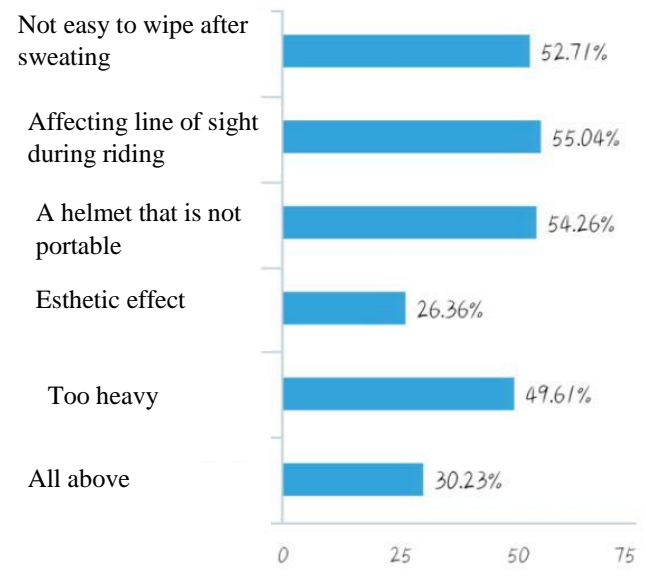

Figure 1 The second sample survey.

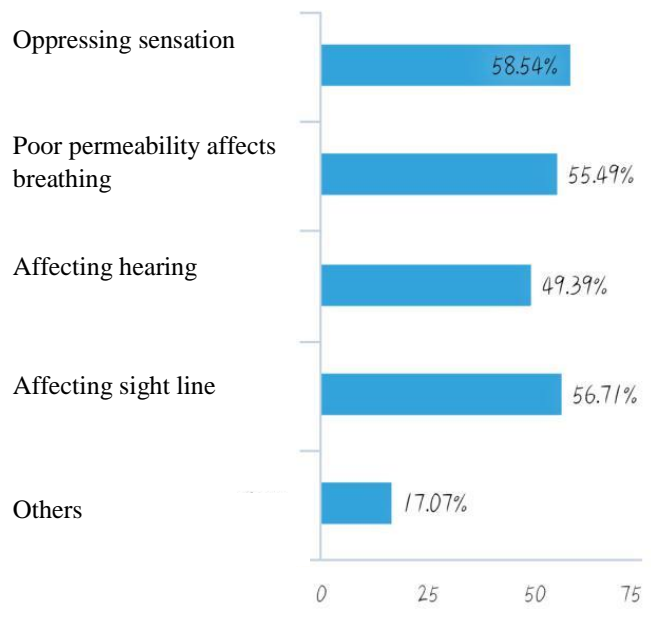

Figure 2 The third sample survey.
"Figure 1" and "Figure 2" show two sample surveys conducted according to the response of the masses. The results show that poor air permeability, bulky material and audio-visual distractions explain the uncomfortable experience. It is also the reason why some people do not want to wear safety helmets or cannot wear them correctly. Further investigation shows that with the continuous improvement of science and technology, people also hope that protective helmets can be more intelligent while being beautiful and comfortable, such as advanced road warning system, positioning service and communication system, and removable transformation, etc. The first two items account for the largest proportion. If there is a new product about helmets, $51.16 \%$ are willing to try it. Nowadays, when people buy helmets, most people consider safety, comfort and price in order.

\section{PATH ANALYSIS TO HELP IMPLEMENT POLICIES EFFECTIVELY}

\subsection{Perfecting the Laws and Regulations}

As of October 1, 2020, a total of 23 local laws and regulations have stipulated the wearing of electric bicycle helmets. Legal constraints and moral guidance can enhance citizens' awareness of safety and law-abiding, so it is fully reasonable to pass legislation requiring e-bike riders to wear helmets correctly. Here are three suggestions for the improvement of local laws and regulations in Jiangsu Province:

The road traffic safety regulation of Shantou special economic zone: "experiential punishment", should be employed as reference, including at least one hour of traffic counseling or at least two hours of attending a traffic safety study or watching traffic safety videos. [1:46] In addition, the intersection traffic flow and traffic police personnel arrangement should also be taken into consideration to appropriately enhance the mobility and flexibility of the police force, increase the severity of punishment, including the installation of special electronic monitoring probes, etc., in order to reduce the fluke mentality of people riding electric bicycles.

Measures of Zhejiang Province for the Administration of Electric Bicycles should also be adopted as reference to strengthen the management of online rental electric bikes. It clearly stipulates that Internet electric bicycle rental enterprises shall provide safety helmets with their vehicles. If they 
fail to provide safety helmets in accordance with the provisions, the traffic administrative department of the public security organ shall order them to make corrections, impose a warning, and impose a fine. In addition, the city's traffic management department should also formulate relevant management regulations for the express delivery industry, which requires more people with electric bikes, so that delivery platforms, businesses and delivery personnel can emphasize the importance of wearing helmets when riding e-bikes.

National standard helmet production regulations should be established as soon as possible. At present, the helmet industry associations in Yueqing, Zhejiang, Shenzhen and other places have independently issued standards for local industry groups to guide the production and quality testing of enterprises. Only by establishing national standards as soon as possible can people guarantee the production of high-quality electric vehicle helmets.

\subsection{Strengthening Publicity and Popularization}

The publicity and popularization mentioned here mainly includes two aspects. The first is to popularize the importance of wearing safety helmets and relevant local laws and regulations to the public. The second is to educate the public about how to properly wear safety helmets.

\subsubsection{Strengthening Publicity and Popularization in the Community}

Community, as a place of residents' life and activities, is one of the important ways to popularize safety knowledge. Therefore, the community should serve as the medium of "uploading and distributing" local laws and regulations, popularizing the laws and regulations and safety knowledge in time. In the community, the practice of printing and distributing propaganda color pages, pushing prompt messages, setting relevant content in the community public board, and posting prompt messages can be adopted to strengthen publicity and popularization.

\subsubsection{Using "We Media" to Strengthen Publicity and Popularization}

In recent years, We media communication has become a way gradually recognized and loved by the society. All regions can follow the example of
Nanjing police, and strengthen publicity and popularization by making relevant educational videos to the society with the help of media, innovating to carry out helmets for free via Douyin Live, and making short videos.

\subsubsection{Strengthening School Education}

Students, as the direct objects of school education, also have the ability of family education and dissemination. Schools can strengthen the publicity and popularization by organizing parents and students to participate in activities as A Complimentary Helmet for Studying the Law, and lottery for free helmet "Lucky Helmets with You".

\subsection{The Positive Role of Enterprises Should Be Brought into Play}

The policy has received a lot of positive responses from enterprises. For example, Yadi took the lead in responding to the "One Helmet and One Belt", Xibaoda shared bikes were also equipped with helmets. In addition, Alibaba 1688 platform will provide support such as product search weighting, scene marketing promotion and personalized recommendation to targeted buyers for helmet spot products that meet the national regulations. "The product must meet the requirements of national standards and must be in stock (delivery within 72 hours)" is also emphasized. Patrol detachment under of Handan Municipal Public Security Bureau, People's Insurance Company of China, and caring enterprises including Tianjin Talang Technology Co., Ltd. Handan Branch and Qixiaobao Hebei Intelligent Technology Co., Ltd., jointly launched a large-scale publicity campaign "One Helmet and One Belt Ensures Safety", highlighting the role of enterprises.

Therefore, major enterprises actively respond to the call of the state, playing a leading role in the demonstration and providing preferential treatment and convenience to the general public. Whether it is possible to "wear" a helmet for sharing electric vehicles, whether it is possible to get a helmet for free for purchasing electric vehicles, how to promote the safe travel of internal employees and how to innovate the functions of employee safety assurance, etc., are still to be solved. Key industries such as express delivery and food delivery should strengthen internal supervision, consciously make helmets a part of travel, guide the general public to wear helmets voluntarily, and 
encourage the whole people to develop safe and civilized traffic habits.

\subsection{Optimizing Helmet Structure and Promoting Smart Helmet}

The personalized recommendation of targeted buyers carried out by Alibaba 1688 platform is worth learning. The orientation of service objects and products can help citizens buy comfortable products and indirectly promote the effective implementation of policies. Although "tailor-made" for everyone is not practical, manufacturers can strive to provide consumers with desirable products through regular research from the material, style, quality, sales models. At the same time, in-depth research should also be carried out on "how to promote mass customization of modular smart helmets". [6]

"Intelligent" is the most intuitive manifestation of scientific and technological progress. In fact, smart helmets have been designed and applied in some fields, such as mine tunnels, construction sites and other industrial fields, and the emergency system of smart helmets based on 5G has also been gradually developed. [7] The survey results show that advanced road warning system, location service and communication system are the most desirable features for citizens. In an emergency, intelligent helmets can more effectively ensure the safety of citizens by virtue of their various functions. However, product price, technology cost and other factors undermine its wide application in the short term, which requires further exploration and trail.

\section{CONCLUSION}

Policy background, policy implementation of Nanjing, social research results and effective paths are analyzed in this paper to explore the implementation path of wearing safety helmet when riding electric bicycles. Based on social research and reference literature, four effective approaches including improving laws and regulations, strengthening publicity and popularization, giving play to the positive role of enterprises, optimizing helmet structure, and promoting smart helmet, are observed, in a bid to provide a more scientific direction for policy implementation and management.

However, the above paths, far from comprehensive, are restricted by many factors in implementation. For example, the positive role played by enterprises involves government policies, enterprise management structure, etc., which requires more detailed exploration in the future.

\section{AUTHORS' CONTRIBUTIONS}

Siyi Jiang analysed data and wrote the manuscript, and Qi Liu wrote the manuscript and contributed to revising.

\section{REFERENCES}

[1] Luo Fangfang, Yu Hong. Discussion on local legislation of electric bicycle helmet wearing in China [C]. Beijing: Road Traffic Management, 2020: 44-46. (in Chinese)

[2] Cao Zhilong. Research on the generating mechanism of public policy under the framework of multi-source model analysis [J]. Rural Economy and Science-Technology, 2020,31(11): 322-336. (in Chinese)

[3] Song Yunong, Ma Weiwei, Shen Jian, et al. Analysis study of the relationship between helmet wearing and casualties of electric vehicle drivers [C]. Chinese Journal of Urban and Rural Enterprise Hygiene, 2020: 7-9. (in Chinese)

[4] Li Ying. "One helmet and one belt" strictly implement the hot purchase of electric helmets [C]. China Quality Promotion, 2020: 94-95. (in Chinese)

[5] Jiang Debin. Electric vehicle helmets are in urgent need of national standards $[\mathrm{N}]$. China Audit Report, 2020-10-19(06). (in Chinese)

[6] Zhang Wenjuan, Ma Weitao, Lan Haisheng. Application of smart helmet system based on $5 \mathrm{G}$ in emergency communication [J]. New Technology. New Business, 2020, 40 (07): 45 48. (in Chinese)

[7] Yuan Wenshan. Research on Modular Smart Helmet Design for Mass Customization [J]. West Leather. 2020. (in Chinese) 